Recherches en didactique des langues et des cultures

Les cahiers de l'Acedle

15-2 | 2018

Quelles médiations en didactique des langues et des cultures?

\title{
Penser la médiation dans une perspective diversitaire
}

Médier et/ou dés-accorder?

\section{Emmanuelle Huver}

\section{OpenEdition}

Journals

Édition électronique

URL : http://journals.openedition.org/rdlc/2964

DOI : $10.4000 /$ rdlc. 2964

ISSN : $1958-5772$

Éditeur

ACEDLE

\section{Référence électronique}

Emmanuelle Huver, «Penser la médiation dans une perspective diversitaire », Recherches en didactique des langues et des cultures [En ligne], 15-2 | 2018, mis en ligne le 02 juin 2018, consulté le 19 septembre 2019. URL : http://journals.openedition.org/rdlc/2964 ; DOI : 10.4000/rdlc.2964

Ce document a été généré automatiquement le 19 septembre 2019.

\section{(c) $(1) \Theta \Theta$}

Recherches en didactique des langues et des cultures is licensed under a Creative Commons AttributionNonCommercial-NoDerivatives 4.0 International License 


\title{
Penser la médiation dans une perspective diversitaire
}

\author{
Médier et/ou dés-accorder?
}

Emmanuelle Huver

\section{Introduction}

1 Cet article poursuit une double visée: d'une part, il s'agira de situer le terme de médiation et ses modalités de conceptualisation et de traitement dans les sciences humaines et sociales (SHS), en s'intéressant plus particulièrement au domaine de la didactologie /didactique des langues (DDdL); d'autre part, il s'agira de poser quelques principes pour une conceptualisation «diversitaire » de la médiation. J'entends ici par "DDdL diversitaire » une DDdL qui fait le pari de "prendre la diversité au sérieux » (Huver et Bel 2015), en l'envisageant non pas seulement comme une thématique à inclure dans la DDdL (thématique de la diversité linguistique et culturelle notamment), mais comme un principe épistémologique et (donc) politique, de fait transversalement agissant et altérant, à différents niveaux de réflexion et d'intervention (conception de la recherche, conception de l'intervention, conceptualisation des notions, etc.). Au-delà de la conception d'outils et de dispositifs, les travaux qui s'inscrivent dans cette orientation cherchent à envisager les implications de cette altération pour la DDdL dans son ensemble, la thèse défendue par l'équipe de recherche à laquelle j'appartiens étant que cette inclusion radicale (au sens propre du terme - jusqu'aux racines) de la diversité en DDdL suppose de recourir plus particulièrement aux apports de la philosophie phénoménologique - herméneutique ${ }^{1}$.

Dans une première partie de mon propos, je proposerai donc quelques éléments d'histoire et de théorisation de la notion en SHS pour mettre en évidence les convergences et les désaccords, voire les conflits, qui en ressortent. Dans une deuxième partie, je m'intéresserai plus particulièrement au domaine de la DDdL, pour envisager les manières dont ces convergences et ces désaccords s'y manifestent (ou non) et les problématiques spécifiques que pose la notion dès lors qu'il est question 
d'appropriation et de langues. Dans une dernière partie, je reviendrai sur quelques notions qui m'apparaissent comme particulièrement capitales pour une compréhension « diversitaire » de la médiation.

\section{Poser les enjeux de la réflexion : éléments d'histoire et de théorisation dans les SHS}

3 Dans cette première partie, on partira de l'étymologie du terme et de ses définitions usuelles dans différents domaines des SHS pour expliciter, dans un second temps, les points qui font / ont fait débat.

\section{Origines et acceptions usuelles}

\section{Etymologie du terme}

4 L'étymologie du terme médiation est intéressante en ce qu'elle pose d'emblée les enjeux d'un débat qui, nous le verrons, n'en finit pas de se poser, au plan de la recherche comme de l'intervention, autour du statut et de la place du conflit. Liquète (2010) et Pruvost (2012) soulignent en effet que le terme repose, dès son origine sur une ambiguïté. En effet, en français, il apparait au $13^{\text {ème }}$ siècle sur la base du latin mediare, qui renvoie à l'idée de division, de séparation entre 2 parties. Ce n'est que plus tardivement (vers le $16^{\text {ème }}$ siècle) que le terme évolue vers l'idée de conciliation (sur la base de la racine latine medius, qui renvoie à la "manière d'être entre »), originellement dans une acception théologique, renvoyant au lien et à l'écartèlement entre l'homme et Dieu. C'est enfin au $19^{\mathrm{ème}}$ siècle, du fait d'un détour par la diplomatie, que le terme prend le sens que nous lui connaissons actuellement: le médiateur, en diplomatie, étant celui qui est chargé d'établir le dialogue pour obtenir un accord, le terme médiation a ainsi fini par désigner, dans le vocabulaire courant, «un intermédiaire facilitateur ".

5 Mais l'histoire du terme ne s'arrête pas là : dans les années 1970 en effet, il fait florès aux Etats-Unis, pour désigner un "mode généralisé de gestion de la vie sociale cherchant à anticiper les risques de conflit par l'entremise d'un tiers» (Six, 2001). C'est aussi à cette époque que la médiation devient une profession, d'abord aux EtatsUnis dans les années 70, puis en Europe dans les années 80 (ibid.). On voit donc ici comment un terme qui renvoyait initialement à l'idée de séparation (et donc, possiblement, de conflit) en est venu à désigner son inverse, puisque qu'il s'agit désormais de concilier des positions conflictuelles, voire d'éviter (anticiper) les conflits.

\section{Héritages et implications pour le présent}

Si je m'arrête ici quelques instants sur l'origine de la notion de médiation, c'est moins pour en proposer une histoire exhaustive que pour mettre en évidence, à grands traits, les principaux éléments dont on retrouve la trace en DDdL. Ceux-ci, on le verra, sont en effet souvent présentés de manière assertive et unilatérale. Ce détour par l'histoire me permet donc de montrer le caractère construit d'affirmations qui se présentent usuellement comme "naturelles» et "sans histoire» (dans les deux sens du terme, dans la mesure où cette évacuation de l'histoire est également une évacuation des antagonismes que celle-ci comporte, un moyen d'éviter de «faire des histoires », donc). 
Je m'appuierai ici notamment sur l'historicisation proposée par Caune (1999), laquelle puise ses sources chez Foucault (dans Les mots et les choses plus particulièrement), pour retenir plus particulièrement deux traits: l'héritage platonicien et l'héritage pragmatiste.

7 L'héritage platonicien est au fondement même de la notion de médiation : l'allégorie de la Caverne repose en effet sur l'idée que nos rapports avec le monde ne sont pas immédiats, mais nécessairement médiés, notamment par le langage. Cette idée à fondement platonicien du langage comme médiateur par excellence est une idée très usuellement reprise dans les conceptualisations de la notion, on y reviendra (cf. infra 3 ème partie).

8 Par ailleurs, Caune montre bien comment la médiation, en tant que notion, s'est constituée à partir du milieu du $19^{\text {ième }}$ siècle, avec la constitution des sciences sociales.

Avec la constitution des sciences sociales, dont l'objet de connaissance se définit à partir du travail et des richesses produites (l'économie), de l'organisation des groupes dans la collectivité (la sociologie) et du langage (la linguistique), se développe l'idée que les éléments de l'objet ont entre eux des relations internes qui assurent une fonction à la totalité examinée. Et c'est l'articulation entre, d'une part, une logique interne aux phénomènes et, d'autre part, le rapport à un ensemble organisé plus large que l'idée de la médiation se fera jour. (Caune, 1999 : 185).

Dans le sillage de ce constat, il insiste sur l'importance à donner aux théories du signe dans la construction du concept de médiation. $\mathrm{Si}$, en l'occurrence, il penche plutôt pour les théories sémiotistes du signe (ou plutôt pour une convergence entre sémiologie et herméneutique), il se livre néanmoins à une critique acerbe de la conception actuellement dominante de la médiation, qu'il considère comme un dévoiement de cet héritage pragmatiste. Selon lui en effet, la médiation est aujourd'hui asservie à une idéologie communicationnelle ${ }^{2}$ et instrumentalisée comme outil de l'action, du fait d'une part de la centration sur des techniques visant l'efficacité et la transparence des échanges, et en raison, d'autre part, d'une conception de l'acteur social comme agent d'une action rationnelle en vue de la réalisation d'un but. Ces analyses pourraient largement être rapprochées de certaines critiques développées en DDdL à l'encontre des approches didactiques actuellement dominantes (communicatives / actionnelles), y compris pour réfléchir la médiation, critiques développées à partir d'arguments relatifs à la survalorisation des techniques (d'enseignement et de communication), à la visée d'efficacité des échanges et à la conception de l'humain comme d'abord rationnel (Castellotti 2017 ; Prieur 2017 ; Anderson 2016).

\section{Eléments définitoires}

La notion de médiation est mobilisée dans différents domaines des SHS et/ou domaines professionnels (par exemple, et sans exhaustive, le droit, la culture, la santé, la communication -notamment interculturelle-, l'éducation, la diplomatie, etc.). Au-delà de cette forte diversité, on peut toutefois relever deux tendances transversales, que l'on retrouve d'ailleurs aussi en DDdL. D'une part, sa popularité à la fois dans le domaine de la recherche et dans le domaine de l'intervention en fait une notion pour laquelle ne peut pas ne pas se poser l'articulation entre ces deux. D'autre part, sa diffusion transdisciplinaire et transprofessionnelle s'est accompagnée d'une importante dilution, ou, à tout le moins, d'une banalisation, au point que le terme en est venu à désigner tout processus de mise en relation : la question se pose ainsi de savoir ce qu'il pourrait 
signifier et dans quelles perspectives théoriques (voire épistémologiques) il s'inscrit, en DDdL plus particulièrement.

L'empan de la notion et la diversité de ses définitions sont tels qu'en proposer une définition unifiée tient de la gageure, d'autant que les définitions varient selon les domaines et les époques. On peut, pour commencer, s'appuyer sur les deux citations suivantes, qui ont une certaine valeur paradigmatique :

Recherche du lien entre l'énonciateur et le récepteur. Ce lien s'établit grâce à une tierce personne et/ou un ensemble de techniques, d'outils, de messages ou d'interfaces accompagnant le récepteur afin de lui faciliter la compréhension par la construction de sens (...) [et] faire se rencontrer des intentions de communication. Liquète $(2010: 11)^{3}$

La médiation associe et concilie deux parties jusqu'alors distantes, se méconnaissant, voire en conflit, en rétablissant la communication. (ibid. : 11)

Un processus de communication éthique reposant sur la responsabilité et l'autonomie des participants dans lequel un tiers, impartial, indépendant et neutre, sans pouvoir décisionnel ou consultatif, avec la seule autorité que lui confèrent les médieurs, favorise (...) le rétablissement du lien social, la prévention ou le règlement de la situation en cause. (Guillaume Hofnung, 2009 : 72)

Ces définitions ne peuvent certes pas être mises tout à fait sur le même plan: les premières relèvent des sciences de la communication et visent essentiellement à remettre en question le modèle binaire émetteur-récepteur, au fondement du schéma conventionnel de la communication. La dernière relève quant à elles du domaine du droit, et renvoie au projet d'éviter les recours juridictionnels. Il en ressort néanmoins les points suivants, qui sont autant de fils constituant la trame de la notion telle qu'elle est actuellement conceptualisée, notamment en DDdL :

- Tiers : il s'agit là d'une caractéristique souvent présentée comme fondatrice de la médiation en tant qu'elle la différencie d'autres notions proches, comme relation, par exemple, qui serait duelle et non ternaire ${ }^{4}$. Ce tiers peut être une personne - c'est alors ce qui fonde la professionnalisation du domaine (i.e. le métier de médiateur); mais il peut également être un outil, une technique - ce qui ouvre la porte à différentes formes de technicisation du processus de médiation. Est également souligné le caractère «neutre » du tiers, qui ne doit donc ni déformer ni intervenir ni s'impliquer, mais simplement "transmettre» (communiquer).

- Production : le fait de concevoir la médiation comme une transmission de sens par un tiers place la notion du côté de la production. Il s'agit en effet de produire du sens intelligible par les parties en présence, sens préexistant qu'il s'agit donc de ne pas déformer, en vertu du principe de neutralité. La production de ce sens passe par ailleurs par un outillage, c'est-àdire des techniques et des procédures - plusieurs auteurs faisant ainsi référence à la nécessité de respecter des étapes formalisées (par exemple: Ben Mrad 2002; BonnaféSchmitt dir. 2003 ; Guillaume-Hofnung 2009 ; Milburn 2002 ; Six 2001). Cette « technologie du social », au fondement des métiers de la relation (dont le métier de médiateur), «s'appuie sur le développement des sciences humaines et sociales, (...) [c'est-à-dire] en somme sur l'idée qu'on peut objectiver l'être humain » (Demailly, 2008: 58). Elle permet ainsi, par l'homogénéisation des procédures matérielles, de formaliser la relation à des fins d'efficacité de l'action. C'est également la nécessité de ces procédures qui légitime le fait que le professionnel puisse revendiquer une position d'expert. On voit donc ici en quoi pôle de la production, homogénéisation, techniques et positionnement d'expert sont indéfectiblement liés, et s'enracinent dans l'héritage pragmatiste susmentionné (cf. par exemple la visée d'efficacité de l'action). 
- Consensus : on l'a vu dans les définitions citées ci-dessus, la visée de la médiation est de prévenir ou de résoudre les conflits ${ }^{5}$. Par ailleurs, Ben Mrad (2002) insiste sur la nature consensualiste des normes mobilisées dans les pratiques de médiation et Guillaume-Hofnung sur la nature contractuelle de la rencontre entre les deux parties, ce contrat étant lui-même de nature consensualiste. Le consensus représente donc une valeur transversale de la médiation, à la fois visée, objectif et moyen de celle-ci.

\section{Une notion au cœur de différentes controverses} deux, particulièrement révélateurs des manières dominantes de penser la notion, notamment en DDdL. Ainsi, dans un premier temps, je m'intéresserai aux enjeux liés à sa démocratisation et à la conception diffusionniste qui en découle, en faisant un détour par le domaine de la médiation culturelle et la manière dont celle-ci est abordée par J. Caune $(2006,1999)$. Dans un second temps, je traiterai des liens entre conceptions de la médiation et modèles (politiques) de société, en m'appuyant sur les réflexions critiques développées par de Briant et Palau (1999) dans le domaine de la médiation juridique.

\section{Démocratisation, production, diffusion}

Dans le domaine culturel, J. Caune développe l'idée que la médiation est usuellement pensée sous l'angle de la production, ce qui se caractérise notamment par le fait que prédominent une conception expressive de l'art et, par conséquent, de la médiation artistique. Dans ce cadre, l'œuvre d'art est d'abord envisagée en tant que pouvoir d'expression; la médiation artistique est alors prioritairement définie au niveau institutionnel (i.e. du point de vue de l'offre culturelle) et sous l'angle de la création (il s'agit alors de produire, créativement, des objets ou des performances artistiques). Dans ce cadre, le médiateur est un passeur d'œuvres (et, en général, d'œuvres «consacrées ») et sa mission est d'en favoriser l'accès auprès des publics (et notamment des publics dits en difficulté ou éloignés de la culture). Il s'agit alors d'une part de préparer les conditions de la rencontre entre le public et l'œuvre et, d'autre part, de développer une politique permettant de réduire la coupure entre culture populaire et culture de l'élite. C'est notamment cette conception diffusionniste de la médiation qui est à l'œuvre en France depuis l'après-guerre, sous l'impulsion de la politique culturelle de Malraux, fondée sur une distinction entre le domaine de l'éduction, dont la visée, rationnelle, est d'enseigner les œuvres et le domaine de la culture, dont la visée, sensible, est de faire aimer les œuvres (éducation artistique etc.). l'œuvre produite et réfléchit à sa diffusion institutionnelle auprès des publics), une médiation qu'il nomme esthétique et qui relève prioritairement de l'expérience individuelle. Dans ce cadre, la médiation vise à permettre l'expérience esthétique, qui ni ne se limite à la jouissance de l'œuvre esthétique (mais est d'abord compréhension de soi), ni n'exclut le social (il s'agit de faire, aussi, l'expérience d'un sens partagé). Le médiateur a alors pour mission de susciter une diversité d'espaces et de situations favorisant l'expérience esthétique de chacun.

16 Cette analyse me semble particulièrement intéressante pour sa résonnance avec la DDdL : celle-ci est à mon sens également essentiellement envisagée sous l'angle de la 
production plus que de la compréhension, et sous l'angle de l'action plus que de l'expérience, comme en témoignent par exemple la centration des méthodologies généralement préconisées sur la communication, dans ses dimensions de production, et sur l'action (cf. perspective actionnelle). La médiation s's'inscrit donc en toute vraisemblance également dans cette logique - nous y reviendrons.

\section{Professionnalisation et circulation des notions : enjeux politiques}

Le second point de controverse que je souhaite aborder touche à la problématique des idées, des valeurs et des projets sous-jacents aux notions et à la manière dont ces idées / valeurs / projets circulent sans nécessairement être perçus, ni interrogés, a fortiori lorsque les notions sont prioritairement appréhendées sous leur angle procédural et/ ou praxéologique (vs épistémologique / historicisé). Cette technicisation des notions conduit en effet à leur naturalisation, puisqu'elles n'apparaissent dès lors plus comme construites, c'est-à-dire, fondamentalement, le fruit de choix épistémologiques et donc - politiques, historiquement situés, quant à des conceptions de l'homme et de la société.

Premier détour nécessaire : un détour historique. Six (2001) explique que la médiation (en tant que secteur professionnel) naît aux Etats-Unis dans les années 1970, à partir d'une tradition de fait proprement états-unienne de méfiance vis-à-vis de l'Etat, ce qui a pour conséquence que les conflits sont gérés par des centres relevant de la philanthropie privée (appelés " conflict resolution centers »). Elle est ensuite importée en Europe «à partir de cette problématique américaine (...), alors qu'il n'y a pas de tradition, en France, de philanthropie privée » (ibid. : 150-151).

Lorsque la médiation (en tant qu'activité professionnelle) est ensuite importée en Europe dans les années 1980, ces différentes orientations circulent avec elle, ce que l'on retrouve par exemple très nettement dans les théorisations de la médiation juridique. En effet, les chercheurs qui ont théorisé cette dernière (Guillaume Hofnung, Bonafé Schmitt, Six, notamment) explicitent tous qu'elle vise à éviter une approche trop judiciarisée du droit (en privilégiant autant que possible la médiation plutôt que le recours en justice), dans une perspective de gouvernance partagée. Entre aussi en compte, plus largement, la crise de l'autorité dans nos sociétés occidentales contemporaines, qui a pour conséquence la remise en question de formes de régulations "par le haut ", au profit de relations plus horizontales et d'une définition plus « consensualiste» (vs impositive) des normes.

Deuxième détour, théorique. La notion de médiation est usuellement définie comme une expertise (cf. également Demailly 2008) exercée par un tiers indépendant (vs mandaté) et neutre, selon une "méthode très précise » (Guillaume Hofnung, $2009: 83$ ), de manière à garantir un positionnement impartial et équilibré. Le médiateur doit être un professionnel, dont le rôle est de mettre en relation des intersubjectivités pour que ces dernières arrivent à un accord librement consenti. La logique sous-jacente est donc à la fois particulariste (à chaque situation sa solution) et contractualiste (les "médiés » doivent arriver à un consensus, notifié dans un contrat, qui a force de référence et d'engagement): cette définition est actuellement très largement dominante et imprègne l'ensemble du monde social.

De Briant et Palau (1999) se sont attachés à développer une critique virulente de cette définition de la médiation, en rappelant d'abord qu'elle n'est pas la seule possible au 
prétexte qu'elle serait la seule à être nommée ainsi. Ils rappellent notamment que d'autres formes de médiation ont toujours existé, même si elles n'étaient pas nommées comme telles. Ces médiations, qu'ils appellent «médiations traditionnelles» (ou «médiations innommées », en opposition aux « nouvelles médiations » ou « médiations nommées $\left.»^{6}\right)$, s'inscrivent dans une logique universaliste, en ce qu'elles renvoient à des lois et à des autorités reconnues préalablement à l'acte de médiation et porteuses de valeurs communes. Elles sont mises en œuvre par des non-professionnels ${ }^{7}$, qui ne sont pas neutres, mais au contraire engagés pour la reconnaissance de ces lois, légitimes parce que porteuses d'universel et, de ce fait, garantes d'égalité. L'activité du médiateur est donc d'abord une activité politique, avant que d'être une méthode et, en ce sens, elle est fondatrice du lien social.

Les "nouvelles médiations" se posent explicitement comme une alternative aux médiations traditionnelles, considérées comme exerçant une contrainte illégitime et relevant d'une conception verticale descendante de la définition des normes. De Briant et Palau (1999: 84), quant à eux, soulignent la "parfaite ambiguïté » de la notion: « manière de donner de la souplesse aux institutions", mais aussi, à l'inverse, "produit de professionnels $d u$ droit, des experts qui en dehors de tout véritable contrôle démocratique, justifient l'existence de normes non par une idée du Bien ou parce qu'elles seraient l'expression de la volonté générale, mais par un rapport de forces mesuré lors des conciliations ». Ce faisant, c'est, selon eux, la vie politique toute entière qui se trouve transformée sur le modèle de la médiation sociétale, qui se substitue progressivement à celui de la décision républicaine. Le politique ne consiste plus en la construction d'un cadre de principes et d'un programme, mais revêt une dimension pragmatique, où il s'agit d'abord de trouver des solutions (techniques, donc) qui rassembleront le plus grand nombre. Enfin, cette conception contractualiste et intersubjectiviste de la médiation, telle que défendue par les théoricien.ne.s des "nouvelles médiations", est parfaitement compatible avec, voire fonde, un modèle socio-économique néo-libéral. Les propos de Guillaume Hofnung (1995 : 98) ne souffrent ici d'aucune ambiguïté :

«La médiation constitue un choc en retour des empiètements de l'Etat Providence sur la vie privée.»

Certes, on reconnait dans l'idéaltype de la "médiation traditionnelle » une certaine forme de républicanisme jacobin, ou, à tout le moins, centraliste, qui voit dans la diversité un danger de fragmentation du social (de Briant et Palau parlent d'ailleurs explicitement des risques de communautarisme que comportent les «nouvelles médiations $\left.»^{8}\right)$. Bien que ce positionnement renvoie à un projet tout à fait opposé au projet diversitaire que je défends, il me semble toutefois gênant d'éluder les critiques émises par ces chercheurs autour du projet social et du modèle de société favorisé par les "nouvelles médiations". En effet, même si cette controverse touche a priori d'autres secteurs professionnels, avec donc d'autres enjeux et problématiques, je crois que nous ne pouvons pas ne pas nous poser la question des effets de l'importation (géographique et disciplinaire) de cette conception intersubjective et contractualiste de la médiation dans notre domaine.

Les propos d'Aden (2012: 271), par exemple, illustrent les fortes porosités entre les différents secteurs d'intervention concernés par la médiation :

Il est essentiel également de ne pas perdre de vue le fait que l'émergence de la notion de médiation dans le CECR est le fruit d'un tressage serré entre une philosophie humaniste, un imaginaire démocratique et une éthique néolibérale des marchés. (...) Dès lors que la circulation des personnes et des biens devient une 
norme, la gestion de la médiation linguistique s'impose comme une nécessité socioéconomique et constitue une priorité dans les politiques linguistiques et interculturelles.

De même, Anquetil (2009: 19), en faisant explicitement référence à la dichotomie de de Briant et Palau, estime qu'il est nécessaire de "prendre ses distances avec les médiations traditionnelles ", de manière à ne plus

«mettre en relation l'individu avec une transcendance ou un universel en basant son autorité sur un système de valeurs partagées (...) mais [à] créer les conditions d'un lien social rompu entre deux individus ou communautés dont les intérêts paraissent divergents »

Ainsi, en définitive, on peut se demander si cette importation souvent non (ou en tout cas peu) questionnée ne participe pas, indirectement et implicitement :

- de la psychologisation des rapports sociaux: la médiation telle que définie par North et Piccardo (2016) participe en effet déjà pleinement de ce processus, de par son ancrage dans un paradigme psycho-cognitiviste (émergentiste), qui en favorisant une entrée biologisante, évacue de fait les enjeux sociaux, politiques et historiques liés à la notion ;

- de la technicisation de la médiation. Demailly (2008) note avec justesse que la professionnalisation, dans les métiers de la relation, va de pair avec l'apparition de technologies du social, visant l'efficacité de l'action par la mise en œuvre de procédures (outils, dispositifs). Le parallèle n'est-il pas facilement réalisable avec l'enseignement, dont il est attendu qu'il soit "efficace", ce qui a pour conséquence que la recherche devrait s'envisager sous l'angle des méthodes rendant possibles (voire garantissant) cette efficacité et que la formation des enseignants consisterait alors dans le fait de les former à la bonne utilisation de ces méthodes?

- d'une forme d'hygiénisme social liée à la judiciarisation des relations sociales : le fait que les «nouvelles médiations» se donnent pour objectif principal de régler ou de prévenir les conflits (de manière contractualiste) n'est-il pas symptomatique d'une société fondée sur la prévention des risques, voire sur l'idée d'un « risque 0 », dont on connaît déjà les effets délétères ?

Ainsi, à l'instar de ce que font de Briant et Palau (et même si, encore une fois, je ne partage que très partiellement leurs analyses, ou plutôt le projet politique à partir duquel ils les développent), n'y aurait-il pas une certaine pertinence à repolitiser, dans notre domaine, la réflexion sur la notion de médiation? Repolitiser étant entendu ici comme le fait de faire apparaître et d'amener à argumenter les choix (politiques, éthiques, épistémologiques, mais aussi ontologiques, voire métaphysiques) qui fondent les positionnements et les théorisations, ce qui suppose d'introduire du débat et du conflit, là où la plupart des ouvrages généralistes présentent la notion de manière très lissée, en éludant ou en minimisant cette controverse?

C'est en tout cas ce que je m'attacherai à faire dans la suite de cet article, en m'intéressant d'abord aux manières dont la notion est conceptualisée dans notre domaine (en lien, notamment, avec les points évoqués dans cette première partie) pour, ensuite, proposer quelques éléments de mise en débat, au plan épistémologique plus particulièrement. 


\section{Et en DDdL ? Entre circulations et spécificités} nombre important de colloques et de publications sur ce sujet depuis une dizaine d'années9. Elle est fortement montée en puissance depuis les années 1990, sous l'influence conjuguée de plusieurs phénomènes (Tapia 1999) : questionnements relatifs à la socialisation et à l'intégration des populations d'origines culturelles diverses ; sensibilité accrue à l'hétérogénéité culturelle; ouverture des frontières (et, plus largement, avènement d'entités supranationales - dont le Conseil de l'Europe, qui occupe une place centrale dans la production et la diffusion de travaux sur la médiation), évolution des mentalités, désormais plus demandeuses de gestion concertée et participative, etc. Elle a fait l'objet de différentes conceptualisations, qui témoignent de sa vitalité tout en laissant paraître un certain nombre d'éléments récurrents, plus ou moins problématisés et que je vais m'efforcer de mettre ici en évidence.

\section{Médiation et théorie médiationnelle}

Il est peut-être intéressant de rappeler qu'en DDdL, la médiation a été traitée dans les années 1970 sous un angle désormais largement oublié. Galisson et Coste (1976) indiquent en effet que les «théories médiationnistes » ont tenté, tout en restant dans un cadre behavioriste, de prendre en compte, grâce à la notion de "processus intermédiaires", certains phénomènes comme l'intériorité du sujet, jusqu'ici «ignorés» des behavioristes car non directement observables. Dans le cadre de la réflexion sur les contacts de langues, la théorie médiationnelle a conduit à distinguer deux modes d'acquisition d'une L2: selon qu'il y a transfert des processus médiationnels de L1 vers L2 (il y a alors "système linguistique composé») ou construction de processus médiationnels spécifiques à la L2 (il y a alors système linguistique coordonné). Galisson et Coste rappellent également que ce second mode d'acquisition est au fondement des méthodes dites directes (i.e. qui visent à faire acquérir les L2 de manière «naturelle ", en se fondant sur des mécanismes identiques à ceux qui ont conduit à l'acquisition de la L1). Si cette théorisation a, depuis, fait l'objet de nombreuses critiques, il n'en demeure pas moins qu'elle renvoie à une problématique récurrente de la DDdL, autour de la prise en compte de la L1 problématique élargie à la prise en compte de la diversité linguistique, fondamentale dès que lors que l'on s'intéresse à la médiation.

\section{Médiation et plurilinguisme}

31 Depuis une vingtaine d'années et dans l'acception que nous lui connaissons aujourd'hui, la médiation a été popularisée par les travaux du Conseil de l'Europe, notamment le Cadre européen commun de référence pour les langues (CECR) et le Cadre de Référence pour les Approches Plurielles des Langues et des Cultures (CARAP). Le CECR considère la médiation comme une activité langagière à part entière, au même titre que la réception, la production et l'interaction. Il définit le médiateur comme un « intermédiaire entre des interlocuteurs incapables de se comprendre en direct », dans des situations orales ou écrites (interprétariat, traduction, résumé et reformulation de 
textes dans la même langue lorsque le texte original est incompréhensible pour son destinataire). Un certain nombre de traits définitoires usuels de la notion sont repris, notamment :

- le fait que le médiateur «n'a pas à exprimer sa pensée mais doit simplement jouer le rôle d'intermédiaire » (Conseil de l'Europe, $2001:$ 71)

- le caractère très largement procédural de la médiation (distinction de différentes stratégies à déployer pendant l'activité de médiation (planification, exécution, évaluation, remédiation).

L'intérêt ici est d'ancrer la didactique dans une perspective explicitement plurilingue, visant, donc, à prendre en compte la diversité des langues (et des répertoires langagiers) dans l'apprentissage d'une langue particulière (à l'inverse d'approches communicatives plus ou moins revisitées- par la perspective actionnelle par exemple mais maintenant la focalisation sur la langue « cible » uniquement, voire continuant à véhiculer l'idée de la nécessité ou de l'efficacité du «bain linguistique ( $\mathrm{cf}$. par exemple Aden $2012: 278$ ).

Cette définition est ensuite précisée dans le CARAP, où la compétence de médiation est désignée comme ce " qui fonde toutes les " mises en relation », entre langues, entre cultures et entre personnes" (Candelier et al. 2007, p. 39). La problématique de la diversité linguistique et culturelle est ici centrale, voire incontournable, non seulement dans la définition elle-même mais aussi du fait que la compétence de médiation est intégrée dans une «macro-compétence à gérer la communication linguistique et culturelle en contexte d'altérité $»^{10}$.

\section{Perspectives politiques, anthropologiques et critiques}

Le CECR et le CARAP constituent ainsi des jalons centraux pour la visibilité et la diffusion de la notion de médiation en DDdL, et pour son arrimage aux problématiques plurilingues. Les travaux développés autour de $\mathrm{G}$. Zarate vont quant à eux contribuer à ancrer la notion dans un courant théorique explicité et argumenté et, ce faisant, à développer un point de vue critique sur la médiation telle que définie dans le CECR. Dans ces travaux, la médiation est en effet inscrite dans une sociologie de l'altérité, telle que développée par Bourdieu plus particulièrement, et mise en lien par ailleurs avec l'anthropologie et la pragmatique interculturelle.

Certaines des caractéristiques principales de la définition usuelle sont reprises par les auteurs, notamment le fait que le médiateur :

- est un intermédiaire restaurant une communication brouillée ;

- doit être dûment mandaté ;

- a pour objectif la résolution des conflits.

Mais ces apports théoriques permettent aussi de mettre en discussion certaines des options affirmées dans le CECR, autour de deux points plus particulièrement. D'une part, la neutralité du médiateur, en ce qu'elle repose sur la conception de «l'entre-deux [comme] espace neutre, "simple», dont la maitrise repose sur une technicité de type linguistique » (Zarate 2003, coord. : 241). A l'inverse de cette conception "pacifiée » de la société, Zarate et les chercheurs associés à son projet de recherche souhaitent centrer leur attention sur les rapports de force et les dysfonctionnements. 
D'autre part, les théories évoquées supra permettent de critiquer des conceptions souvent essentialisantes et réifiantes de la culture, qui fondent des approches différentialistes de l'interculturel, selon laquelle, dans la médiation, c'est «la formulation d'éléments statiques et explicatifs qui peut résoudre les problèmes de communication » (Liddicoat, $2015: 3)^{11}$

\section{Elargissements}

Plus récemment, la notion de médiation a été élargie sous deux angles, qui s'avèrent en fait être complémentaires : d'une part sous l'angle de l'enaction et, d'autre part, sous l'angle d'un projet d'amplification du CECR visant notamment à élaborer des niveaux et des descripteurs pour la compétence de médiation.

C'est notamment sous l'impulsion de J. Aden que la médiation se trouve conceptualisée dans un cadre de référence enactif, selon lequel « la médiation linguistique et culturelle [est] à la fois une compétence socle de l'interaction sociale et un espace de potentialisation où le sens se négocie dans un translangager " (Aden 2012 : 268). Aden situe ses travaux dans la continuité des principes introduits par le CECR. Celui-ci opère en effet selon elle une transition "entre une logique de l'acquisition et une logique de l'expertise» (Aden, ibid.), cette dernière pouvant être définie comme la capacité à mettre en lien le familier et le nouveau dans des contextes inédits. A ce titre, la «didactique de l'expertise ou de l'efficience", introduite par le CECR, "constitue un marche-pied vers les modèles émergentistes » (ibid.).

Le projet d'amplification du CECR, entamé en 2014, marque de ce point de vue une nouvelle étape, en ce qu'il s'ancre dans des théories de référence explicitées ${ }^{12}$, en l'occurrence autour de la dimension neuro-psycho-physiologique de l'apprentissage des langues, suivant en cela l'invitation d'Aden. L'inscription dans les sciences cognitives est ainsi manifeste, voire prégnante, dans les études qui servent d'appui au projet d'amplification (cf. notamment North et Piccardo, 2016). Le rapport de Coste et Cavalli (2015) est quant à lui moins explicite et sans doute plus prudent sur ce point (cf. leur article dans le présent volume), mais il n'en demeure pas moins que la médiation y est définie comme à la fois relationnelle et cognitive, ces dimensions étant envisagées à parité, voire comme dans un rapport de subordination du relationnel au cognitif, ce qui ouvre la voie au traitement cognitiviste qui en est ensuite fait dans le projet lui-même.

41 La survalorisation de l'ancrage «psycho » de la médiation (au détriment de son ancrage «socio ») n'est toutefois pas sans conséquence: la biologisation des dynamiques à l'œuvre dans l'apprentissage (et dans l'enseignement) des langues participe de leur naturalisation et, en définitive, de leur technicisation (cf. sur ce point Simard 2004) - ce qui renvoie aux questions génériquement posées à la fin de la partie précédente. Le problème n'est pas, de mon point de vue, que ces théorisations (qu'on peut globalement qualifier de communicatives et socioconstructivistes) existent - le problème est plutôt celui de leur actuelle domination ${ }^{13}$, au point qu'il apparaît comme difficile de penser hors d'elles, comme si elles constituaient un horizon indépassable, une sorte de « fin de l'histoire ".

42 Aden (2012) proposait de penser la médiation dans un cadre théorique unifiant; c'est donc plutôt à la tentative inverse qu'il me paraît important de se consacrer. C'est pourquoi, dans cette dernière partie de mon propos, je m'attacherai à proposer quelques réflexions visant à dés-unifier ces cadres pour mettre en débat certaines 
orientations qui restent pour l'instant souvent peu discutées (voire peu perçues comme discutables - au sens de : à discuter).

\section{Vers quelques éléments de mise en débat}

43 Je reprendrai ici trois notions (comprendre, langue et conflit) dont les modalités de mobilisation pour traiter de la médiation me sont apparues comme constituant des points de consensus relativement unanimes dans le domaine de la DDdL. L'objectif ici sera de les mettre en discussion au regard des orientations «diversitaires" développées au sein de mon équipe de recherche (cf., sur ce point, l'introduction de cet article).

\section{Comprendre}

Caune (1999) faisait état, pour la médiation culturelle, de la survalorisation de la production au détriment de la réception et, plus particulièrement, de ce qu'il nomme l'expérience esthétique. De même, Liquète $(2010: 21)$ considère que le médiateur est un "spécialiste de la transmission". On retrouve cette même tendance en DDdL, où l'objectif usuellement visé est celui de la production, ne serait-ce que dans la manière dont est usuellement conçue une séquence d'enseignement ${ }^{14}$.

Pour ce qui concerne la médiation, le terme comprendre est récurrent dans les définitions qui en sont proposées. Liddicoat (2015:4) insiste ainsi sur le fait que la médiation consiste à "comprendre divers autres", par «le développement de compréhensions partagées entre participants à la communication ${ }^{15}$. Pour autant, si l'on y regarde de plus près, il s'agit moins de mettre l'accent sur le comprendre luimême que sur les moyens de produire des énoncés qui puissent être compris. Comprendre est alors conçu comme un "se faire comprendre " (faire comprendre son intention de communication), le comprendre étant en fait lui-même compris au filtre du pôle de la production :

"L'interprétation du signe passe par une compréhension de l'intentionnalité de celui qui l'émet » (Caune, $1999: 191$ )

[la médiation vise à] "améliorer et faire se rencontrer des intentions de communication » (Liquète $2010: 10$ )

"Quant au médiateur, ne serait-il pas l'un et l'autre, celui qui évalue en fonction de la loi et celui qui produit dans l'échange, pour l'autre et pour soi.» (Lévy, 2003 : 22)

"On doit ainsi enseigner une "production médiatrice", ou art de contrôler sa production langagière en langue maternelle afin d'en faciliter la compréhension. » (Anquetil, $2009: 21$ )

Cette conception "productiviste » de la médiation va de pair avec une conception très majoritairement communicativiste de celle-ci (et, plus largement, de la DDdL) : il est ainsi particulièrement frappant de constater que la médiation est systématiquement mise en lien avec la notion de communication (élargie, dans certains cas, à ses dimensions culturelles et interculturelles - cf. par exemple Zarate coord. 2003). Cette focalisation sur la communication contribue à ancrer la DDdL - et donc la médiation dans une visée et/ou un cadre de référence pragmatiste, et notamment dans les théories de l'action (cf. l'actuelle suprématie de la perspective actionnelle ${ }^{16}$ ). Caune (2006) prône ainsi, pour la médiation culturelle, une pragmatique de la culture fondée sur des références théoriques liées à la philosophie analytique (Frege, Austin, etc.) - de 
même que Hunyadi (1995) élabore, au plan philosophique, la théorisation d'une "pragmatique de la médiation" fondée sur les travaux de Wittgenstein et de Bouveresse.

Cette tendance est également visible en DDdL, tant, par exemple, chez G. Zarate coord. (2003), qui cite explicitement la pragmatique comme un de ses cadres de référence, que chez Liddicoat (2014), qui développe tout un article sur les liens entre la pragmatique et la médiation interculturelle dans l'apprentissage des langues.

L'alliage de la production et de la communication aboutit ainsi, de fait, à s'intéresser en priorité à la production de messages (et de sens) compréhensibles, au détriment d'une interrogation sur les manières dont d'autres comprennent - la question de la réception étant ici inséparable de ses dimensions diversitaire (diversité des réceptions) et altéritaire (les sens des autres m'étant toujours en partie "étrangers", voire incompréhensibles). Lévy (2003: 12) craint que « loger la didactique des langues dans le cadre de l'incompréhension" conduise à sa "dramatisation ». Mais ne peut-on pas considérer, à l'inverse, qu'une didactique des langues sourde aux réceptions, y compris parfois dérangeantes et conflictuelles, est une didactique des langues sourde à sa propre histoire et aux histoires des personnes qui contribuent à la faire vivre ${ }^{17}$ ?

Que signifierait alors envisager la DDdL prioritairement du point de vue de la réception? Il ne s'agit pas, bien sûr, de concevoir ici la compréhension comme un simple décodage de signes, mais de l'envisager dans le cadre plus large du " comprendre », un comprendre fondé sur la diversité des expériences et des vécus (phénoménologique, donc) et sur leur conflictualité (herméneutique, donc). Pour le dire rapidement, concevoir la médiation sous l'angle du comprendre suppose de la concevoir d'abord comme un traduire ${ }^{18}$, dont le sens est toujours provisoire et toujours pluriel, à la fois parce qu'il est fondamentalement expérientiel et parce que " comprendre, c'est toujours comprendre autrement » (Gadamer 1996).

Or, ce point de vue est finalement très peu abordé en DDdL, tant au plan de l'intervention (où la traduction occupe actuellement une place mineure et déconsidérée, a fortiori dans une perspective autre qu'actionnelle et communicative) qu'au plan de la recherche (où la réflexion didactique à partir de la pluralité des rapports aux langues et des projets avec les langues reste peu développée, de même qu'est peu conceptualisée, au plan épistémologique, ce que serait, pour notre domaine, une recherche véritablement " compréhensive »).

51 De cette première exploration découlent donc deux interrogations, elles-mêmes très en lien avec la question de la médiation : d'une part, une interrogation autour de la notion de langue, dès lors que celle-ci n'est plus d'abord conçue sous l'angle de la production et de la communication, et, d'autre part, autour de la notion de conflit, qui prend, dans les approches phénoménologiques-herméneutiques (désormais $\mathrm{PH}$ ), un statut à la fois central et particulier, possiblement heuristique pour penser la médiation.

\section{Langue}

Dans les diverses théorisations de la médiation évoquées précédemment, la notion de langue n'est pas (n'est plus) réellement conceptualisée, comme si cette notion n'avait plus besoin d'être réfléchie. En DDdL, on retrouve très massivement le motif de la langue comme moyen de communiquer ${ }^{19}$. Plus largement, et transdisciplinairement, les théorisations de la médiation reposent toutes sur l'idée platonicienne évoquée dans la 
première partie selon laquelle le « réel » n'est pas connaissable immédiatement, si bien que toute connaissance passe par des médiations, le langage représentant à cet égard «le médiateur par excellence » (Guillaume-Hofnung 2009, 70). On retrouve la même orientation en DDdL, par exemple dans l'ouvrage de référence que constitue le Dictionnaire de didactique $d u F L E / S$, où il est dit de la médiation qu'elle est "présente d'emblée dans le langage » (Cuq dir., $2003: 163$ ).

La phénoménologie fait ici exception (cf. de Briant et Palau, 1999 : 41) en remettant au centre la notion d'expérience et, plus précisément, en posant une antériorité de droit (et non une antériorité génétique) de l'expérience " muette encore » (Romano 2012) sur la pensée, même si celle-ci est remaniée en profondeur par le langage. Lorsque la phénoménologie (et donc la primauté donnée à l'expérience ${ }^{20}$ ) croise l'herméneutique (et donc la primauté du point de vue de la réception, du comprendre), la théorisation de la notion de langue ${ }^{21}$ s'en trouve radicalement transformée : celle-ci ne peut plus être considérée prioritairement comme un moyen, un outil ou un instrument, mais devient au contraire, un «milieu» (un "être-propre » ${ }^{22}$ ), qui « dépasse infiniment toute intention ${ }^{23}$ que nous pouvons y mettre» (Lacan, cité par Berman $\left.1984: 229\right)$. La langue (et a fortiori la langue étrangère, qui est de ce point de vue paradigmatique) est ainsi d'abord et toujours opacité, incompréhensibilité, d'autant que l'expérience "précède toute interprétation " (Berman, $1984: 271)$ et que, de ce fait, il existe du sens et du « comprendre » en-deçà, au-delà et à travers le langage ${ }^{24}$.

Or, la philosophie analytique (le linguistic turn) a occulté toute cette dimension expérientielle, au sens propre anté-prédicative (Romano 2012), et il n'est donc pas étonnant qu'elle soit également absente de toute la DDdL actuelle, puisque celle-ci se fonde, précisément, sur les théories issues de la philosophie analytique (Austin et Searle, pour ne mentionner que certains des plus cités dans notre domaine).

Une fois ce constat épistémologique posé demeurent deux questions, que l'on peut trivialement formuler ainsi : " qu'est-ce que ça change ? » et « qu'est-ce que ça a à voir avec le projet «diversitaire » évoqué en introduction?». Je ne peux bien sûr pas répondre ici dans le détail à ces questions (cf. pour cela Castellotti 2017), mais tenterai d'expliciter certaines implications du fait de réhabiliter cette dimension expérientielle, en l'occurrence dans la médiation.

Si on considère que la médiation s'enracine dans des vécus expérientiels, la position de neutralité du médiateur n'est, irrémédiablement, plus tenable. La critique ne porte pas ici sur un plan méthodologique, comme le faisait G. Zarate en mettant en question l'entre-deux comme espace neutre, mais sur un plan ontologique, en considérant que l'humain (et ici, singulièrement, le médiateur) ne peut pas ne pas être impliqué dans la situation (en l'occurrence de médiation). Il en va en effet de sa condition d'humain que d'être au monde et aux autres, que de se pro-jeter, existentiellement, dans la relation ; il ne peut pas ne pas avoir de «sentiment de la situation» (David, $2004: 1217)^{25}$. Son travail n'est alors pas d'être un "intermédiaire facilitateur " (figure fréquemment convoquée pour qualifier l'enseignant, dès lors qu'il s'agit de se détacher d'une figure patriarcale et verticalement transmissive - cf. par exemple Anquetil 2009), mais de comprendre, à partir de sa propre projection expérientielle (de son «sentiment de la situation »- en s'impliquant, donc), que d'autres comprennent autrement et comment ils comprennent autrement - effort toujours risqué et pari jamais totalement remporté.

Il s'agit aussi, en tant qu'enseignant de langue(s), de mettre les personnes en situation(s) de vivre (et de réfléchir) des expériences avec les langues. Langue non pas 
comme système qu'il s'agirait de manipuler, ou comme ensemble de règles (pragmatiques) de communication qu'il s'agirait de mettre en scène (pour reprendre la métaphore théâtrale de Goffman et renvoyer, en même temps, aux activités communicatives de type «jeux de rôles » ou « simulations », bien connues en DDdL) dans des activités qui relèvent plus de l'expérimentation que de l'expérienciation ${ }^{26}$. Mais langue en tant que registre de l'être, à la fois « épaisseur de la capacité parlante " ${ }^{27}$ et confrontation à l'altérité.

La didactique des langues étrangères a ici un rôle tout particulier à jouer, en tant qu'expérience de l'altérité (Py, 1992), ou, pour reprendre les mots de Berman « épreuve de l'étranger ». La réflexion que produisent Berman d'une part et Steiner d'autre part, sur la traduction, sont ici particulièrement heuristiques, à la fois en tant qu'illustration de ce que peut signifier l'expérience de l'étranger et en tant que moyen (didactique) de faire vivre cette expérience.

Sans entrer dans les détails, Steiner et après lui Berman ${ }^{28}$ essayent de penser une traduction qui permette de faire l'expérience de «l'altérité des êtres» (Steiner 1998 : 636), ce qui passe par une réhabilitation d'une forme de littéralité (à l'inverse de la recherche d'équivalence, qui cherche à faire oublier la traduction en en évacuant l'effet étrangeant). Outre le fait que cela peut en soi constituer une activité didactique pertinente, dans une visée diversitaire / altéritaire (cf. à ce sujet Castellotti 2017), il me semble que cette réflexion sur la traduction vaut également, mutatis mutandis, pour la didactique des langues. S'approprier une langue, c'est en effet la transformer au filtre de son expérience pour « la faire advenir en propre » (Dastur 2011: 95), c'est y faire entendre, à rebours de conceptions normatives et homogénéisantes de la langue et de son enseignement, donc diversitairement, « la violence du métissage » (Berman 1984 : $16)^{29}$.

\section{Conflit}

60 Cette expression, la "violence du métissage ", n'est à mon sens pas anodine et rend compte à sa façon, de la part intrinsèquement conflictuelle que porte en eux l'enseignement / apprentissage des langues et donc la médiation, dès lors que l'on se place du point de vue de la réception et des projets d'appropriation des personnes ${ }^{30}-$ dès lors donc qu'on envisage ces phénomènes sous un angle diversitaire et, par conséquent, altéritaire. Il s'agit ainsi d'une question de choix, ou, autrement dit, d'une question politique. Si le choix effectué est de "prendre la diversité au sérieux» (comme je le défends ici), j'argumenterai que l'une des conséquences qui en découle est la nécessité de penser autrement - de manière " positive » - le conflit, et ce à l'inverse de ce qui est usuellement soutenu (à savoir que la médiation a pour fonction d'instaurer ou de restaurer du consensus).

«Face aux amoureux de la guerre, ils [les médiateurs] sont des bâtisseurs de paix.»

«La médiation est « un lieu d'espoir (...) pour passer de la discorde à la fraternité. »

61 Ces citations peuvent sembler bien iréniques, voire naïves et «enchantées ». Il n'en demeure pas moins qu'elles ont été écrites par l'un des auteurs les plus cités sur la notion de médiation, J.F. Six (2001: 11 et 13, respectivement) - citations qui témoignent d'un rapport pour le moins défiant vis-à-vis du conflit. Zarate (2003: 176) fait également le constat, en DDdL, d'un « rapport frileux avec la conflictualité ", ce qui est particulièrement évident dans le cas de la didactique de l'interculturel, qui, généralement, occulte ou diabolise le conflit et réifie la langue et la culture pour les 
constituer en cause des conflits constatés (cf. par exemple Debono 2011 et, dans une certaine mesure, Abdallah-Pretceille 1996).

Au-delà de ces sorties parfois caricaturales, la plupart des chercheurs qui réfléchissent la notion de médiation ne rejettent pas le conflit et affirment même son caractère inévitable, en argumentant avec M. Weber (Le Savant et le politique) que certains conflits sont inexpiables, avec G. Simmel (Le conflit) que le conflit est une forme active de socialisation ${ }^{31}$ et avec H. Arendt (Penser l'événement) que l'absence de conflit est une forme de totalitarisme. Lévy et Zarate (2003 : 186) s'inscrivent également dans cette lignée en concluant qu'une institution qui se dote d'un médiateur " dépasse une vision utopique de la société fondée sur le consensus ».

Le consensus reste néanmoins, au moins implicitement, une visée de ces théorisations. Conjointement à la reconnaissance du conflit est en effet développée l'idée que s'il est mal traité, mal géré, il peut devenir destructeur et avoir un cout économique et humain pour la société. Il s'agit donc de savoir traiter, gérer (autrement dit contrôler) le conflit, dans la perspective, au moins comme horizon, qu'il puisse être "aplani » (Hunyadi 1995), notamment par la «radicale procéduralisation » (ibid.) de la médiation (par la méthode et les dispositifs, donc). De même, Guillaume Hofnung (2009: 86) soutient que "si le médiateur joue bien son rôle, les médieurs s'apercevront qu'ils ne mettent pas la même signification dans le mot qui cristallise l'incompréhension $»^{32}$. Il s'agit donc bien par la médiation (et notamment par les méthodes de médiation mises en œuvre), de garantir une transparence de la communication, elle-même garante de la résolution du conflit.

Cette conception contractualiste, donc consensualiste, $\mathrm{du}$ conflit, pose, plus épistémologiquement, la question du statut de la diversité, notamment de la diversité donc de l'altérité - des points de vue dans ces réflexions. Il s'agit à mon sens d'une conception en quelque sorte homogénéisante de la diversité, dans la mesure où l'on postule que le médiateur peut à terme comprendre l'autre (sans prendre en compte le fait que cette compréhension peut être autre / est sans doute autre que celle de la personne elle-même) et que la juxtaposition des points de vue (l'intersubjectivité) permet à terme leur convergence et, ce faisant, une appréhension exhaustive et donc « objective » du monde :

«Intersubjectivity enables a more objective comprehension of the world, i.e., in which different perspectives may converge » (Thirioux et Berthoz, cité par Aden $2012: 277)$.

On retrouve d'ailleurs ce positionnement, didactiquement traduit, dans la théorie de la multiperspectivité développée par Stradling pour l'enseignement de l'histoire.

On est ici dans un univers de pensée bien différent de celui que je postule, dans lequel le sens n'est jamais uniquement ni prioritairement dans les signes, mais dans les expériences : peut-on en effet penser que certains conflits graves - qui engagent des valeurs, des histoires souvent dans le temps long, etc. - puissent être «résolus » en « mettant la même signification dans les mots »?

Pour les courants phénoménologiques-herméneutiques en effet, le conflit a d'abord à voir non pas avec la langue et la communication, mais avec le «concernement » : il concerne les gens (au sens fort du terme) et parce qu'il les concerne (en d'autres termes parce qu'il n'est pas uniquement rationnel), il échappe, au moins partiellement, à leur contrôle (cf. de Robillard, sous presse). Pour le dire autrement, et pour reprendre la pensée gadamerienne, le conflit est d'abord une expérience herméneutique et, à ce titre: est tributaire de l'historicité, tant collective que personnelle; se fonde sur les 
préjugés, conçus comme une condition de la compréhension elle-même; n'est pas contrôlable (c'est " un advenir dont nul n'est maitre ", dit Gadamer, 1996 : 378).

Cette orientation, toute abstraite qu'elle puisse paraitre à ce stade, a pourtant des implications concrètes en matière de DDdL. En effet, si on considère que les histoires et les expériences personnelles et collectives débordent la significativité de la langue, travailler la médiation linguistique et culturelle suppose, prioritairement, de laisser advenir ces expériences, les sens (antéprédicatifs) qu'elles revêtent pour les personnes et les «conflits d'interprétation", pour reprendre les termes de Ricœur, qu'elles provoquent, non pas pour les aplanir mais pour les faire travailler.

Dans cette optique, le conflit (au sens herméneutique) n'est pas un échec, mais un lieu privilégié d'une relation qui engage l'être, au-delà de la simple confrontation d'opinions divergentes à propos de situations sans enjeu. «En se laiss[ant] dire quelque chose par [l'autre]» (Gadamer, 1996 : 384), la personne accepte alors [ou pas !] que soit touché le cœur même de sa compréhension (non uniquement rationnelle, explicite et consciente) du monde : il s'agit pour le médiateur, de s'engager, au risque (et non au danger) du conflit et du prix à payer pour qu'il travaille, en profondeur, la relation, à savoir le prix de l'altération (sociétale, ontologique, etc.) ${ }^{33}$.

\section{Conclusion : implications pour la DDdL}

«La traduction peut fort bien se passer de théorie, non de pensée. Et cette pensée

s'effectue toujours dans un horizon philosophique. » (Berman, 1999: 17)

70 Cette citation de Berman pourrait tout aussi bien valoir pour la DDdL. Ainsi, de fait, ma visée ici n'était pas de proposer une théorisation de la médiation, mais d'«élucider certains présupposés cachés " (Dastur 2004: 50) pour penser la médiation et ses fondements philosophiques.

71 Il ne me reste plus d'espace pour présenter de manière approfondie des exemples d'activités concrètes fondées sur l'orientation que j'ai défendue. Elles existent néanmoins et sont longuement exposées et argumentées, sous différents angles: confrontation d'interprétations conflictuelles de faits historiques "socialement vifs " (Papasaika, ici-même), ateliers " d'écriture transformative » (Lorilleux, 2015), activités autour de la traduction et de la littérature ${ }^{34}$ et, plus largement, des projets d'appropriation des personnes (Castellotti 2017, ainsi que Courtaud, ici-même).

Je conclurai peut-être en insistant sur le fait que ces différentes activités - et les orientations qui les fondent - ont en commun de penser la DDdL, et donc la médiation, comme devant viser, prioritairement, ce qui in-stabilise et " dés-accorde », dans l'idée de "se défaire de l'aspect familier des choses " (P. Valéry). Médier, en classe de langue, consisterait alors non pas à prévenir ou résoudre les conflits, mais à les susciter, en tant que controverse discutante, concernante : contrepied à son acception usuelle et retour à son étymologie initiale. 


\section{BIBLIOGRAPHIE}

Abdallah-Pretceille M., 1996 [1ère éd. : 1986], Vers une pédagogie interculturelle, Paris : Anthropos. Aden J., 2012, « La médiation linguistique au fondement du sens partagé : vers un paradigme de l'enaction en didactique des langues ", Études de linguistique appliquée, n 167, pp. 267-284. http:// www.cairn.info/revue-ela-2012-3-page-267.htm.

Aden J. et Weismann D. (coord.), 2012, « La médiation linguistique : entre traduction et enseignement des langues vivantes ", Études de linguistique appliquée, $\mathrm{n}^{\circ}$ 167, http:// www.cairn.info/revue-ela-2012-3.htm.

Anderson, P., 2016, Une langue à venir. De l'entrée dans une langue étrangère à la construction de l'énonciation. Paris : L'Harmattan.

Anquetil M., 2009, « La médiation en classe de langue », Le français dans le monde, n 361, pp. 19-21. Ben Mrad F., 2002, Sociologie des pratiques de médiation. Entre principes et compétences, Paris : L'Harmattan.

Berman A., 1999, La traduction et la lettre ou l'auberge du lointain, Paris : Seuil.

Berman A., 1984, L'épreuve de l'étranger. Culture et traduction dans l'Allemagne romantique. Paris : Gallimard.

Besse H., 2009, « Pourquoi apprend-on encore le français en tant que langue étrangère ? », Revue japonaise de didactique du français, vol. 4, $\mathrm{n}^{\circ} 1$, pp. 9-25.

Bonafé-Schmit et al. (dir.), 2003, Les médiations, la médiation, Paris : Erès.

Briant V. de et Palau Y., 1999, La médiation. Définitions, pratiques et perspectives, Paris : Nathan.

Candelier M. et al., 2007, CARAP - Cadre de référence pour les approches plurielles des langues et des cultures. Graz : CELV - Conseil de l'Europe.

http://www.ecml.at/mtp2/publications/C4_report_ALC_F.pdf.

Castellotti V., 2017, Pour une didactique de l'appropriation, Paris : Diier.

Castellotti V., 2015, « Diversité(s), histoire(s), compréhension. Vers des perspectives relationnelles et alterdidactiques pour l'appropriation des langues », Recherches en didactique des langues-Cahiers de l'ACEDLE vol. 12 n 1, 293-331, http://acedle.org/old/spip.php?article4381.

Castellotti V., Debono M. et Huver, E., 2017, « Contexte, contextes, contextualisation en DDdL. D'une didactique contextualisée à une didactique diversitaire ", dans Babault S., Bento M. \& Spaëth V., Tensions en didactique des langues : entre enjeu global et enjeux locaux. Bern : P. Lang, pp. 49-76.

Caune J., 2006, La démocratisation culturelle : une médiation à bout de souffle, Grenoble : PUG.

Caune J., 1999, Pour une éthique de la médiation. Le sens des pratiques culturelles. Grenoble : PUG.

Conseil de l'Europe, 2001, Cadre européen commun de référence pour les langues : apprendre, enseigner, évaluer. Paris : Didier.

Coste D. et Cavalli M., 2015, Education, mobilité, altérité. Les fonctions de médiation de l'école. Strasbourg : Conseil de l'Europe.

http://www.coe.int/t/dg4/Linguistic/Source/LE_texts_Source/LE\%202015/Education-MobilityOtherness_FR.pdf. 
Dastur F., 2011, Heidegger et la pensée à venir, Paris : Vrin.

Dastur F., 2004, La phénoménologie en questions : Langage, altérité, temporalité, finitude, Paris : Vrin.

David P., 2004, « Stimmung », in B. Cassin (dir), Vocabulaire européen des philosophies. Paris : Seuil, pp. 1217-1219.

Debono M., 2011, « Pour une pédagogie du conflit en Chine ? », Synergie Chine, $\mathrm{n}^{\circ}$ 6, pp. 127-140. http://gerflint.fr/Base/Chine6/debono.pdf.

Demailly L., 2008, Politiques de la relation. Approche sociologique des métiers et activités professionnelles relationnelles. Villeneuve d'Ascq : Presses Universitaires du Septentrion.

Dortier J.-F. (dir.), 2008, Le dictionnaire des sciences humaines. Paris : Ed. Sciences humaines.

Gadamer H.-G., 1996 [1 1 ère édition 1960], Vérité et méthode. Paris : Seuil.

Galisson R. et Coste D. (dir.), 1976, Dictionnaire de didactique des langues. Paris : Hachette.

Gaussel M. et Reverdy C., 2013, " Neurosciences et éducation : la bataille des cerveaux ». Dossier d'actualité Veille et Analyses IFÉ, n 86. Lyon : ENS de Lyon. http://ife.ens-lyon.fr/vst/DA/ detailsDossier.php?parent=accueil\&dossier=86\&lang=fr.

Gioia de M. et Agresti G. (coord.), 2016, « Médiation et droits linguistiques », Études de linguistique appliquée, $\mathrm{n}^{\circ}$ 181, http://www.cairn.info/revue-ela-2016-1.htm.

Guillaume-Hofnung M., 2009 [1 1 ère édition : 1995], La médiation. Paris : PUF.

Heidegger M., 1958, Essais et conférences. Paris : Gallimard.

Hunyadi M., 1995, La vertu du conflit. Pour une morale de la médiation. Paris : Cerf.

Huver E. et Bel D. (coord.), 2015, Prendre la diversité au sérieux en didactique / didactologie des langues. Altériser, instabiliser : quels enjeux pour la recherche et l'intervention?, Paris : L'Harmattan.

Lévy D., 2003, « Médiation, didactique des langues et subjectivité », in : D. Lévy et G. Zarate coord., « La médiation et la didactique des langues/cultures », Le français dans le monde Recherches et applications, janvier, pp. 10-23.

Levy D. et Zarate G., 2003, « La médiation dans le champ de la didactique des langues et des cultures », in : D. Lévy et G. Zarate coord., « La médiation et la didactique des langues/cultures », Le français dans le monde - Recherches et applications, janvier, pp. 186-189.

Liddicoat, A. J., 2015, « Intercultural mediation, intercultural communication and translation », Perspectives Studies in Translatology, https://www.researchgate.net/publication/ 273514068_Intercultural_mediation_intercultural_communication_and_translation.

Liddicoat, A. J., 2014, « Pragmatics and intercultural mediation in intercultural language learning ». Intercultural Pragmatics, 11(2), 259-277.

Liquète V. coord., 2010, Médiations. Paris : CNRS Editions.

Lorilleux J., 2015, Ecritures transformatives. Quand des élèves allophones deviennent auteurs, ou : de l'appropriation à l'émancipation ?, Thèse de doctorat, Université F. Rabelais - Tours.

Maurer B., 2011, Enseignement des langues et construction européenne. Le plurilinguisme, nouvelle idéologie dominante. Paris : Éditions des archives contemporaines.

Medhat-Lecocq H. et al. (coord.), 2016, Traduction et apprentissage des langues : Entre médiation et remédiation, Paris : Éditions des archives contemporaines.

Milburn P., 2002, La médiation : expériences et compétences. Paris : La Découverte. 
North B. et Piccardo E., 2016, Elaborer des descripteurs illustrant des aspects de la médiation pour le Cadre européen commun de référence pour les langues, Strasbourg : Conseil de l'Europe.

Prieur J.-M., 2017, « L'empire des mots morts. Lisons le CECR comme un cauchemar », in Maurer B. et Prieur J.-M. coord., «La pensée CECR », Travaux de didactique du FLE, n 70, http://revuetdfle.fr/les-numeros/numero-70/21-1-empire-des-mots-morts-lisons-le-cecr-comme-uncauchemar\#sdfootnote1anc.

Pruvost J., 2012, « Avant-propos. La médiation, un maître mot, un mot de maîtres », Études de linguistique appliquée, $\mathrm{n}^{\circ}$ 167, pp. 261-263, http://www.cairn.info/revue-ela-2012-3-page-261.htm.

Py B., 1992, « Acquisition d'une langue étrangère et altérité », Cahiers de l'ILSL, nº 2, pp. 113-126, http://www.unil.ch/webdav/site/clsl/shared/CILSL02.pdf.

Robillard de D., sous presse, «L'arc et la lyre Ou le statut épistémologique du désaccord », in : J. Ravat et A. Wagener coord., La valeur du désaccord, CNRS Editions.

Robillard de D., 2007, « La linguistique autrement : altérité, expérienciation, réflexivité, constructivisme : en attendant que le Titanic ne coule pas ", Carnets d'atelier de sociolinguistique 1, pp. 81-228.

Robillard de D. (dir.), 2016, « Epistémologies et histoire des idées sociolinguistiques », Glottopol, $n^{\circ} 28$.

Romano C., 2012, « Les repères éblouissants. Sur l'antéprédicatif et la phénoménologie du langage. ", P. Cadiot dir., " Linguistique et phénoménologie du langage », La Tribune internationale des langues vivantes, $\mathrm{n}^{\circ}$ 52/53, p. 5-29.

Romano C., 2010, Au cœur de la raison : la phénoménologie. Paris : Gallimard, Folio.

Simard D., 2004, Education et herméneutique. Contribution à une pédagogie de la culture, Laval : Presses Universitaires de Laval.

Six J.-F., 2001 [1 $1^{\text {ère }}$ éd. : 1990]. Le temps des médiateurs. Paris : Seuil.

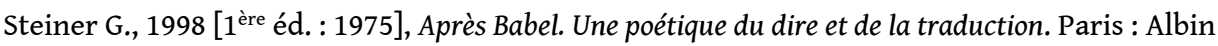
Michel.

Tapia C. coord., 1999, « La médiation interculturelle », Cahiers de Sociologie économique et culturelle, $n^{\circ} 32$.

Zarate G., 2003, « La médiation en situation de tension identitaire », in : D. Lévy et G. Zarate coord., " La médiation et la didactique des langues/cultures », Le français dans le monde Recherches et applications, janvier, pp. 173-184.

Zarate G. coord., 2003, Médiation culturelle et didactique des langues, Strasbourg : CELV - Conseil de l'Europe.

\section{NOTES}

1. Je ne peux développer ici ce point de manière très détaillée : on y reviendra, sans doute encore trop brièvement, dans la dernière partie de cet article, mais le lecteur intéressé pourra se référer à de Robillard dir. (2016) et à Castellotti (2017). Je souligne également que ces différents travaux dont le présent article - sont le fruit d'un intense travail collectif mené par l'EA 4428 Dynadiv, sur le plan à la fois de l'approfondissement d'un certain nombre d'options communes et de l'argumentation dans la controverse - ces deux pans étant indissociablement heuristiques dans l'instauration d'une pensée en propre. Le terme désaccorder, qui figure dans le titre de cette 
contribution, provient d'échanges en vue de la préparation d'un panel pour un colloque portant sur le désaccord (La valeur du désaccord, Angers, 5 et 6 mai 2015) : il apparait notamment dans la présentation d'I. Pierozak, qui considère le chercheur comme un « désaccordeur professionnel ».

2. Caune appelle communication la transmission d'un contenu prédéterminé.

3. Dans l'ensemble des citations de l'article, les soulignements sont de mon fait.

4. Caune (1999) met en évidence les racines théologiques de cette conceptualisation de la médiation. Il l'ancre notamment dans la tradition juive en présentant le rabbi Juda Loeb ben Bezabel (dit Maharal, 1512-1609) comme penseur de la médiation, avec sa figure du Golem, qui symbolise la médiation qui permet à l'homme d'être créature de Dieu tout en étant lui-même créateur. Pour ce qui nous concerne plus directement, cette figure permet également, selon Caune, de symboliser le pouvoir du langage, dont on retrouve une forme contemporaine dans la cybernétique: Wiener établit en effet une relation entre le Golem et la cybernétique, en instituant la communication comme valeur centrale de l'homme et de la société, postulat que l'on retrouve également dans la DDdL contemporaine (cf. infra).

5. Six (1990) complexifie quelque peu la typologie en ajoutant aux médiations préventive et curative, des médiations créatrice (pour susciter de nouveaux liens) et rénovatrice (pour réactiver des liens distendus). Mais cela n'est que de peu d'importance pour mon propos ici.

6. Briant et Palau insistent également sur le fait que l'opposition effectuée est une opposition d'idéaux-types, au sens wébérien du terme (« outil conceptuel (...) destiné à définir les caractéristiques essentielles des conduites humaines ou des institutions sociales. L'idéaltype est une construction intellectuelle qui ne reflète pas une réalité empirique mais permet d'en analyser les composantes »Dortier dir. 2008 : 313).

7. Selon de Briant et Palau, les médiations traditionnelles peuvent être liées à la religion (le Christ comme médiateur du salut), au voisinage (maire, instituteur, médecin, gardien d'immeuble etc. - en ce qu'ils sont, à différents niveaux, dépositaires d'une certaine autorité) ou au politique (représentants politiques ou syndicaux).

8. Positionnement que, dans le domaine de la médiation interculturelle, on retrouve également chez Tapia (1999 : 15) qui indique explicitement que les médiateurs doivent adhérer au modèle « laïc et républicain, autrement dit, à la tradition française » et, pour parer à tout risque de communautarisme, «ne pas recruter les médiateurs interculturels dans les communautés ethniques ou culturelles concernées par les problèmes d'intégration». Cette tension, très française, entre universalisme et diversité, se retrouve d'ailleurs déclinée en DDdL sur le pan des politiques plurilingues (cf. par exemple Maurer 2011).

9. Outre le colloque qui a donné lieu au présent volume, on peut citer par exemple deux colloques qui auront également lieu en 2017 sur la même thématique (l'un à Paris-Est Créteil : Métissages linguistiques et culturels émergents: vers quelles médiations?, l'autre à Rabat: Médiations de la diversité : productions et usages de la culture en société), ainsi que différentes publications récentes : Aden et Weissman coord. 2012 ; de Gioia et Agresti coord. 2016 ; Liddicoat 2015 ; Medhat-Lecoq et al. coord. 2016.

10. La seconde macro-compétence étant la « compétence de construction et d'élargissement d'un répertoire linguistique et culturel pluriel ».

11. "the formulation of static, explanatory elements that can resolve communication problems" (ma traduction). Cette réification est nettement perceptible chez Six (2001: 192) par exemple: «il peut arriver qu'une médiation concerne des individus de cultures différentes (occidentale, asiatique, africaine), ce qui compliquera singulièrement la tâche du médiateur ».

12. Alors que le CECR revendiquait, de ce point de vue, une certaine «neutralité ». Si l'on peut questionner ce positionnement (en ce qu'il relève en fait plutôt de l'implicitation des théories de référence que de leur prétendue «absence»), il n'en demeure pas moins que le fait que ces références deviennent explicites marque un véritable retournement. Celui-ci est redoublé par le fait que ces références relèvent essentiellement des sciences cognitives, alors que le CECR, 
originellement, s'inscrivait plutôt dans les sciences sociales (notamment la sociolinguistique, plus que la psycholinguistique). Sur le débat autour de l'introduction des neurosciences dans les problématiques éducatives, on peut notamment se reporter à l'utile synthèse de Gaussel et Reverdy (2013).

13. sous des formes parfois atténuées et de ce fait pas toujours aisément perceptibles, mais que l'amplification à venir du CECR viendra en outre légitimer et donc renforcer

14. Il s'agit en effet massivement d'organiser le déroulement des activités de la compréhension vers la production.

15. " understand diverse others "; "development of shared understandings between participants in communication " (ma traduction, c'est moi qui souligne).

16. Que l'on retrouve d'ailleurs également légitimée dans les courants de la didactologie qui s'inspirent de l'enaction varelienne, puisque Varela définit les langues comme «fondamentalement une manière de coupler les individus à l'intérieur d'une espèce pour la coordination de l'action" (Varela, in Aden 2012 : 274).

17. Le cas du français me semble ici assez emblématique, pris en tension entre le Charybde d'une langue au lourd passif historique actuellement encore agissant (langue coloniale, langue historiquement imposée sur le territoire français et langue hautement normative et centralisée) et le Scylla de l'imposition d'approches communicatives, en partie pour combattre cette conception normative et homogénéisante de la langue. Ces cas, par ailleurs si différents, ne prennent finalement que peu au sérieux les «projets d'appropriation» (Castellotti, 2017) des personnes, qui peuvent ne relever ni de l'un ni de l'autre, mais être d'un tout autre ordre, qui n'est plus perceptible dès lors que l'on procède par imposition (langagière, méthodologique) a priori, toute contextualisée cette imposition soit-elle (cf. à ce propos: Besse 2009 ou encore Castellotti, Debono et Huver, 2017).

18. Selon les mots mêmes de G. Steiner (1998), «traduire c'est comprendre et comprendre c'est traduire ».

19. Même si certains chercheurs thématisent la question des liens entre langue et identité (comme G. Zarate, par exemple), la visée prioritairement communicative de l'enseignement des langues n'est pas pour autant remise en question.

20. Au sens que lui donne Romano (2001). On peut également renvoyer à cette lumineuse citation d'Heidegger (in Berman, 1999: 16) : "faire une expérience avec quoi que ce soit, cela veut dire: le laisser venir sur nous, qu'il nous atteigne, nous tombe dessus, nous renverse et nous rende autre. Dans cette expression, "faire» ne signifie justement pas que nous sommes les opérateurs de l'expérience: faire veut dire ici, comme dans la locution "faire une maladie", passer à travers, souffrir de bout en bout, endurer, accueillir ce qui nous atteint en nous soumettant à lui ».

21. Ou, plus exactement la «dimension $\mathrm{L}$ » au sens hyperonymique que lui donne de Robillard (2007), subsumant les distinctions entre langue, langage, discours, etc.

22. Selon les termes bien connus d'Heidegger (1958: 227), «l'homme se comporte comme s'il était le créateur et le maitre du langage, alors que c'est celui-ci au contraire qui est et demeure son souverain (...) car au sens propre des termes, c'est le langage qui parle ».

23. On voit donc ici que le statut de l'intention est tout autre que dans les conceptualisations antérieurement citées.

24. Steiner (1998: 262) cite ce mot d'E. Ionesco: «Il est entendu que les mots ne disent rien (...). L'expérience profonde n'a pas de mots. Plus je m'explique, moins je me comprends. Tout n'est pas incommunicable par les mots, bien sûr, mais la vérité vivante ".

25. Expression qui tente de traduire le terme heideggérien de Befindlichkeit: cette traduction a notamment l'intérêt de montrer qu'on est ici épistémologiquement très loin d'un positionnement interprétativiste, en ce que ce dernier postule que l'interprétation est à base prioritairement rationnelle, là où l'expérience antéprédicative renvoie à une saisis immédiate - sur fond culturel, 
donc - du sens (Dastur, 2004) : en l'espèce, avoir un «sentiment de la situation » n'est par exemple pas du tout la même chose qu' « interpréter le contexte».

26. pour reprendre la distinction opérée en anglais entre to experiment et to experience.

27. Par ce terme un peu obscur, Berman (1984) tente de traduire le terme allemand de Sprachlichkeit, qu'il mobilise dans sa tentative de penser (réhabiliter) une traduction littéralisante (cf. infra dans le texte).

28. Avec certaines différences, qui sont de l'ordre de la nuance pour notre propos.

29. Au lecteur qui souhaiterait une illustration un peu plus concrète de ces réflexions, je renvoie aux chapitres consacrés par Steiner et par Berman à Hölderlin, dans lesquels ils exposent, chacun à leur manière, les principes aux fondements de la traduction, telle qu'elle est pensée par le poète allemand, en tant que "qu'exemple le plus violent et le plus délibérément poussé de pénétration et d'annexion herméneutique» (Steiner, 1998: 439). Les traductions «littérales» et de ce fait extrêmement étrangeantes d'Hölderlin avaient en effet pour visée de «forcer les mots contemporains pour en dégager la racine sémantique» (ibid., 440) et, ainsi, faire ressentir au lecteur étranger à la langue (et à l'univers) d'origine de l'œuvre, une forme de "compréhension authentique liée à l'appréhension linguistique et culturelle d'une différence intraitable » (ibid. : 512).

30. Il n'est que de voir les réactions ironiques, hostiles et parfois violentes face à certaines manières de s'exprimer en français et/ou d'apprendre le français (i.e. vécues comme ironiques / hostiles / violentes par les personnes concernées), alors que du point de vue de ces personnes, ces manières de s'exprimer ou d'apprendre constituent des manières singulières de se projeter dans la langue, de se l'approprier, même si cela est très éloigné des normes linguistiques ou didactiques généralement valorisées. Dans mes réflexions (et celles de mon équipe de recherche), il ne s'agit pas seulement de constater ces expressions de la glottophobie (mais aussi de la « didactophobie »!) plus ou moins ordinaire, dans le but de les dénoncer et de lutter contre elles. Il s'agit également de réfléchir ce qui fait (et persiste à faire) homogénéité (en tant que celle-ci est, par définition «altérophobe »), jusque dans le cœur même des SHS.

31. On notera cependant que le conflit, tel que développé par Simmel, vise surtout à légitimer le capitalisme contre le socialisme, ce qui, au fond, est assez cohérent avec les prises de position, de Guillaume Hofnung par exemple, contre l'Etat Providence.

32. On retrouve la même idée que le conflit - l'incompréhension - est essentiellement dans le langage, lorsqu'elle constate que «à la base des malentendus, il y a les mal-dits, les imprécisions du langage par manque de vocabulaire ou par peur. » (ibid. : 90)

33. Je pense ici particulièrement à cette citation de J. Caune (1999: 12), qui met l'accent sur le fait que la médiation est, au fond, une manière d'étouffer le conflit ou, plus exactement, de ne pas vouloir payer le prix de sa « résolution» :

"L'usage indifférencié de la notion de médiation vaut comme symptôme d'une société qui craint de reconnaitre les conflits, recherche les espaces du dialogue et $d u$ consensus et aspire à renouer le tissu social déchiré par le développement incontrôlé de la logique marchande. La médiation déclinée sous ses multiples modalités (...) focalise les attentes de ceux qui redoutent l'élargissement de la fracture sociale mais ne sont pas prêts à envisager le prix de sa réduction".

34. Sur les liens entre DDdL, littérature et altérité, cf. également Prieur (2017). 


\section{RÉSUMÉS}

Cet article poursuit une double visée : il s'agira d'une part de situer le terme de médiation et ses conceptualisations dans les sciences humaines et sociales en général et en didactique des langues en particulier et, d'autre part, de poser quelques principes pour une conceptualisation « diversitaire » de la médiation.

Dieser Artikel verfolgt zwei Ziele : einerseits strebt er dazu ein, den Begriff von Mediation und seine Konzeptualisierungen in den Geisteswissenschaften, und besonders in Fremdsprachendidaktik, zu fassen. Andererseits handelt es sich darum, einige Grundprinzipien für ein « diversitäres » Verständnis dieses Konzeptes vorzuschlagen.

\section{INDEX}

Mots-clés : médiation, diversité, phénoménologie, herméneutique, langue, comprendre, conflit Keywords : mediation, diversity, phenomenology, hermeneutic, language, understand, conflict

\section{AUTEUR}

\section{EMMANUELLE HUVER}

Emmanuelle Huver est professeure des Universités à l'Université F. Rabelais de Tours (EA 4428 Dynadiv). Ses travaux s'inscrivent principalement dans le champ de la didactique / didactologie du FLE/S et des langues. Les thématiques qu'elle explore sont reliées par la problématique transversale de la diversité (des langues, des cultures, des usages de transmission / appropriation) et de ses enjeux, du double point de vue de la recherche et de l'intervention. Ses travaux les plus récents visent à réfléchir les bases d'une didactique / didactologie des langues diversitaire, sur des bases phénoménologiques et herméneutiques 